Article

\title{
Synthesis of New Macrocyclic Polyamides as Antimicrobial Agent Candidates
}

\author{
Osama I. Abd El-Salam ${ }^{1}$, Mohamed A. Al-Omar ${ }^{2,3}$, Ahmed A. Fayed ${ }^{4}$, Eman M. Flefel ${ }^{4}$ and \\ Abd El-Galil E. Amr ${ }^{1,2, *}$
}

1 Applied Organic Chemistry Department, National Research Center, Dokki 12622, Cairo, Egypt

2 Pharmaceutical Chemistry Department, College of Pharmacy, King Saud University, Riyadh 11451, Saudi Arabia

3 Pharmaceutical Chemistry Department, Drug Exploration \& Development Chair (DEDC), College of Pharmacy, King Saud University, Riyadh 11451, Saudi Arabia

4 Photochemistry Department, National Research Center, Dokki 12622, Cairo, Egypt

* Author to whom correspondence should be addressed; E-Mail: aamr1963@yahoo.com.

Received: 17 September 2012; in revised form: 26 November 2012 / Accepted: 28 November 2012 / Published: 6 December 2012

\begin{abstract}
A series of macrocyclic imides and Schiff-bases have been prepared via the cyclocondensation of pyridine-2,6-dicarbonyl dichloride (1) with L-ornithine methyl ester to give the corresponding macrocyclic bisester $\mathbf{2}$. Treatment of $\mathbf{2}$ with hydrazine hydrate gave macrocyclic bisacid hydrazide $\mathbf{3}$, which was used as starting material. Condensation of bishydrazide 3 with diacid anhydrides or aromatic aldehydes in refluxing acetic acid or ethanol gave the corresponding macrocyclic bisimides $\mathbf{4}, \mathbf{5 a}, \mathbf{b}$ and macrocyclic bishydrazones $\mathbf{6 a}-\mathbf{j}$, respectively. The structure assignments of the new compounds were based on chemical and spectroscopic evidence. The antimicrobial screening showed that many of these newly synthesized compounds have good antimicrobial activities, comparable to ampicillin and ketaconazole used as reference drugs.
\end{abstract}

Keywords: macrocyclic polyamide candidates; amino acids; Schiff's base; antimicrobial activity 


\section{Introduction}

Among the different areas of macrocyclic chemistry, the synthesis and complexing properties of azacrown compounds have been a subject of intensive exploration [1-7]. The chemical modification of antibacterial agents in order to generate novel macrocyclic compounds with better therapeutic properties is necessary because of the emergence of multidrug resistant bacteria [8]. On the other hand, peptides rarely function well as drugs due to their low bioavailability and rapid degradation within cells [9]. In our previous work, we reported the synthesis of some macrocyclic candidates from the reactions of dipicolinic acid with amino acids and their biological activity screening [10-14]. In particular, 2,6-peptidopyridines exhibited a general ionophoric potency [15] and were used for inventing novel thiocyanate-selective membrane sensors [16]. Recently, some new heterocyclic steroidal and macrocyclic derivatives have been studied as 5 $\alpha$-reductase inhibitors, antiviral and anti-tumor agents [17], aromatase and quinone reductase-2 inhibitors [18], anti-inflammatory [19], anticonvulsant [20] and antimicrobial agents [21,22]. In view of these observations and in continuation of our previous work in macrocyclic chemistry, we have now synthesized some new macrocyclic derivatives containing pyridine and amino acid moieties. Some of the synthesized compounds were screened for their antimicrobial activity compared to the reference drugs ampicillin and ketaconazole.

\section{Results and Discussion}

\subsection{Chemistry}

In our previous work, a series of chiral macrocyclic compounds were synthesized using macrocyclic bishydrazide derivative 3 [13], which was obtained from the corresponding ester 2 according to the published procedure $[23,24]$ (Scheme 1).

Scheme 1. Synthetic pathway for starting compound 3.

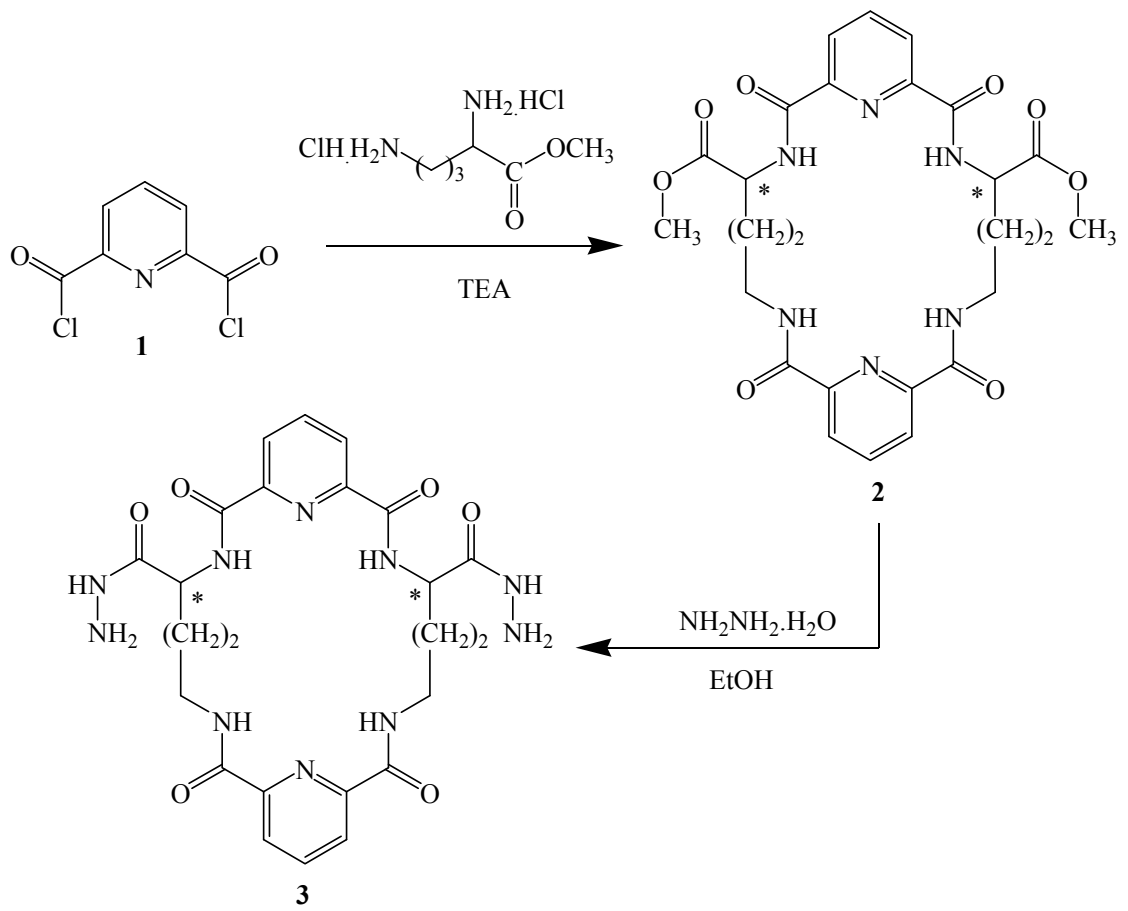


Condensation in refluxing acetic acid of hydrazide $\mathbf{3}$ with selected acid anhydrides, namely 1,8-naphthalic anhydride, phthalic anhydride or 2,3,4,5-tetrachlorophthalic anhydride, afforded the corresponding tricyclobisdiimide derivatives 4 and 5a,b, respectively (Table 1). Additionally, in light of the aforementioned biological interest in hydrazone derivatives [25-27], compound 3 was condensed with aromatic aldehydes in refluxing ethanol to afford the corresponding 4,20-di[oxo(substituted phenyl)-carbohydrazonylmethyl)-3,8,16,21,27,28-hexaaza-2,9,15,22-tetraoxotricyclo$\left[3,21,1,1^{10,14}\right]$ octacosa-1(26),10,12,14,23,25-hexene macrocyclic bishydrazones $\mathbf{6 a - j}$ (Scheme 2 and Table 1). The structures of newly synthesized compounds $\mathbf{4}, \mathbf{5 a}, \mathbf{b}$ and $\mathbf{6 a - j}$ were confirmed by their IR, ${ }^{1} \mathrm{H}-\mathrm{NMR},{ }^{13} \mathrm{C}-\mathrm{NMR}$ and mass spectra.

Scheme 2. Synthetic pathway for compounds $\mathbf{4}, \mathbf{5 a}, \mathbf{b}$ and $\mathbf{6 a}-\mathbf{j}$.

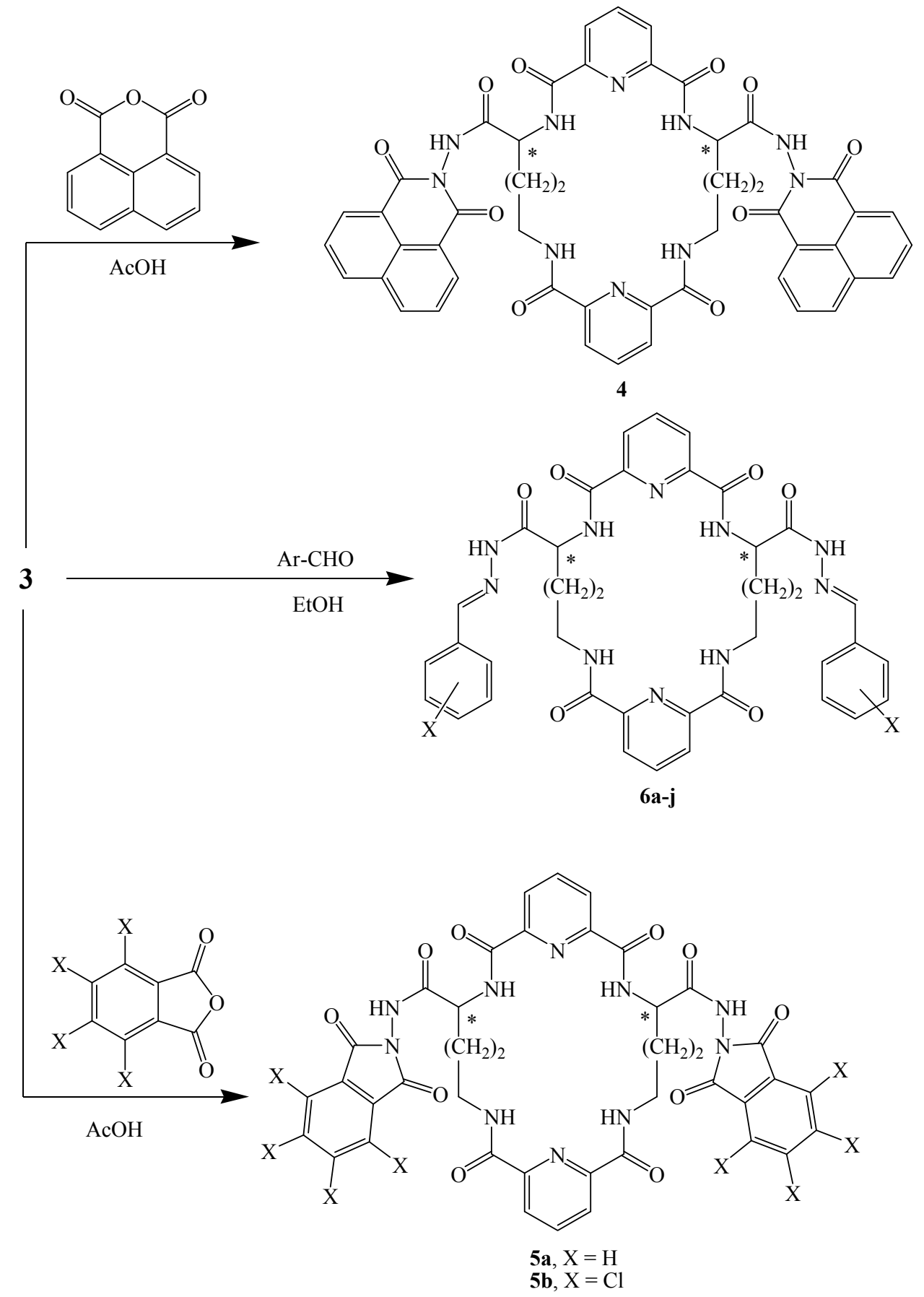


Table 1. Melting points, crystallization solvents, yields, molecular formulae and molecular weights of compounds $\mathbf{4}, \mathbf{5 a}, \mathbf{b}$ and $\mathbf{6 a}-\mathbf{j}$.

\begin{tabular}{cccccc}
\hline $\begin{array}{c}\text { Comp. } \\
\text { No. }\end{array}$ & $\mathbf{X}$ & $\begin{array}{c}\text { M.p. } \\
\left({ }^{\circ} \mathbf{C}\right)\end{array}$ & $\begin{array}{c}\text { Cryst. } \\
\text { Solv. }\end{array}$ & $\begin{array}{c}\text { Yield } \\
(\mathbf{\%})\end{array}$ & $\begin{array}{c}\text { Molecular Formula } \\
\text { (Mol. Wt. }\end{array}$ \\
\hline $\mathbf{4}$ & - & $276-278$ & $\mathrm{DMF} / \mathrm{H}_{2} \mathrm{O}$ & 65 & $\mathrm{C}_{48} \mathrm{H}_{38} \mathrm{~N}_{10} \mathrm{O}_{10}(914.87)$ \\
$\mathbf{5 a}$ & $\mathrm{H}$ & $243-245$ & $\mathrm{DMF} / \mathrm{H}_{2} \mathrm{O}$ & 72 & $\mathrm{C}_{40} \mathrm{H}_{34} \mathrm{~N}_{10} \mathrm{O}_{10}(814.75)$ \\
$\mathbf{5 b}$ & $\mathrm{Cl}$ & $296-298$ & $\mathrm{DMF} / \mathrm{H}_{2} \mathrm{O}$ & 88 & $\mathrm{C}_{40} \mathrm{H}_{26} \mathrm{C}_{18} \mathrm{~N}_{10} \mathrm{O}_{10}(1090.31)$ \\
$\mathbf{6 a}$ & $\mathrm{H}$ & $178-180$ & $\mathrm{EtOH} / \mathrm{Ether}$ & 85 & $\mathrm{C}_{38} \mathrm{H}_{38} \mathrm{~N}_{10} \mathrm{O}_{6}(730.77)$ \\
$\mathbf{6 b}$ & $3-\mathrm{Br}$ & $232-234$ & $\mathrm{MeOH}$ & 79 & $\mathrm{C}_{38} \mathrm{H}_{36} \mathrm{Br}_{2} \mathrm{~N}_{10} \mathrm{O}_{6}(888.56)$ \\
$\mathbf{6 c}$ & $4-\mathrm{Br}$ & $254-256$ & Dioxane & 87 & $\mathrm{C}_{38} \mathrm{H}_{36} \mathrm{Br}_{2} \mathrm{~N}_{10} \mathrm{O}_{6}(888.56)$ \\
$\mathbf{6 d}$ & $2,6-\mathrm{Cl}_{2}$ & $198-200$ & EtO & 68 & $\mathrm{C}_{38} \mathrm{H}_{34} \mathrm{Cl}_{4} \mathrm{~N}_{10} \mathrm{O}_{6}(868.55)$ \\
$\mathbf{6 e}$ & $3,4-\mathrm{Cl}_{2}$ & $188-190$ & $\mathrm{EtOH} / \mathrm{Ether}$ & 78 & $\mathrm{C}_{38} \mathrm{H}_{34} \mathrm{Cl}_{4} \mathrm{~N}_{10} \mathrm{O}_{6}(868.55)$ \\
$\mathbf{6 f}$ & $2-\mathrm{Cl}-6-\mathrm{F}_{\mathbf{6}}$ & $168-170$ & $\mathrm{AcOH} / \mathrm{H}_{2} \mathrm{O}$ & 84 & $\mathrm{C}_{38} \mathrm{H}_{34} \mathrm{Cl}_{2} \mathrm{~F}_{2} \mathrm{~N}_{10} \mathrm{O}_{6}(835.64)$ \\
$\mathbf{6 g}$ & $4-\mathrm{CH}_{3}$ & $155-157$ & $\mathrm{EtOH} / \mathrm{H}_{2} \mathrm{O}$ & 82 & $\mathrm{C}_{40} \mathrm{H}_{42} \mathrm{~N}_{10} \mathrm{O}_{6}(758.82)$ \\
$\mathbf{6 h}$ & $2-\mathrm{OCH}_{3}$ & $210-212$ & $\mathrm{AcOH} / \mathrm{H}_{2} \mathrm{O}$ & 90 & $\mathrm{C}_{40} \mathrm{H}_{42} \mathrm{~N}_{10} \mathrm{O}_{8}(790.82)$ \\
$\mathbf{6}$ & $4-\mathrm{OCH}_{3}$ & $216-218$ & $\mathrm{EtOH} / \mathrm{H}_{2} \mathrm{O}$ & 80 & $\mathrm{C}_{40} \mathrm{H}_{42} \mathrm{~N}_{10} \mathrm{O}_{8}(790.82)$ \\
$\mathbf{6 j}$ & $3,4,5-\left(\mathrm{OCH}_{3}\right)_{3}$ & $235-257$ & $\mathrm{AcOH} / \mathrm{H}_{2} \mathrm{O}$ & 75 & $\mathrm{C}_{44} \mathrm{H}_{50} \mathrm{~N}_{10} \mathrm{O}_{12}(910.92)$ \\
\hline
\end{tabular}

\subsection{Antimicrobial Testing}

The newly synthesized compounds have been tested for their preliminary antimicrobial activity against the following microorganisms: Gram-positive bacteria, represented by Bacillus subtilis (NRRL B-543) and Staphylococcus aureus (NRRL B-313), Gram-negative bacteria, represented by Escherichia coli (NRRL B-558) and fungi, represented by Candida albicans (NRRL Y-477) and Aspergillus niger (NRRL Y-3). The results are summarized in Table 2.

Table 2. Antimicrobial activities of new synthesized compounds $\mathbf{4}, \mathbf{5 a}, \mathbf{b}$ and $\mathbf{6 a}-\mathbf{j}$.

\begin{tabular}{|c|c|c|c|c|c|}
\hline \multirow{3}{*}{$\begin{array}{l}\text { Comp. } \\
\text { No. }\end{array}$} & \multicolumn{5}{|c|}{ Inhibition zone (mm) } \\
\hline & \multicolumn{2}{|c|}{ Gram +ve bacteria } & \multirow{2}{*}{$\begin{array}{c}\text { Gram -ve bacteria } \\
E . \text { coli }\end{array}$} & \multicolumn{2}{|c|}{ Fungi } \\
\hline & B. subtilis & Staph. aureus & & C. albicans & A. niger \\
\hline 3 & 1.75 & 1.55 & 0.80 & - & 1.65 \\
\hline 4 & 1.55 & 1.60 & 0.80 & 0.65 & 1.60 \\
\hline $\mathbf{5 a}$ & 1.35 & 1.75 & 0.60 & 0.75 & 1.90 \\
\hline $5 b$ & 1.80 & 1.48 & - & - & 1.65 \\
\hline $6 a$ & 1.20 & 1.70 & - & - & 1.85 \\
\hline $6 \mathbf{b}$ & 1.45 & 1.45 & 0.80 & - & 1.60 \\
\hline $6 c$ & 0.90 & 1.30 & - & 0.65 & 1.70 \\
\hline 6d & 1.80 & 1.25 & 0.60 & - & 1.80 \\
\hline $6 e$ & 1.75 & 0.85 & - & - & 1.75 \\
\hline $6 f$ & 1.30 & 1.50 & - & 0.70 & 2.05 \\
\hline $6 \mathrm{~g}$ & 0.85 & 1.30 & 0.75 & 0.65 & 1.95 \\
\hline $6 \mathrm{~h}$ & 1.80 & 1.25 & 0.70 & 0.60 & 1.65 \\
\hline $6 \mathbf{i}$ & 1.70 & 1.20 & 0.75 & - & 1.55 \\
\hline $\mathbf{6 j}$ & 1.60 & 1.45 & 0.70 & 0.55 & 1.75 \\
\hline Ampicillin & 1.15 & 1.30 & 0.75 & - & - \\
\hline Ketaconazole & - & - & - & 0.80 & 2.30 \\
\hline
\end{tabular}


From the data it appears that some of the synthesized compounds have modest antimicrobial activity. Except for $\mathbf{6 c}, \mathbf{6 g}$ (against $B$. subtilis) and $\mathbf{6 d}, \mathbf{6 e}, \mathbf{6 h}, \mathbf{6} \mathbf{i}$ (against $S$. aureus) the activities of tested compounds against these Gram-positive bacteria are slightly higher than that of the refence drug ampicillin. The activities of compounds $3,4,5 \mathbf{a}, \mathbf{b}, \mathbf{6 b}, \mathbf{6 d}$, and $\mathbf{6 g}-\mathbf{j}$ against the Gram negative bacterium $E$. coli were all similar to the activity of the reference drug, while compounds $\mathbf{5 b}, \mathbf{6 a}, \mathbf{6 c}, \mathbf{6 e}$ and $\mathbf{6 f}$ are inactive against the same organism. In addition, the compounds tested against fungal organisms ( $C$. albicans and A. niger) had lower activities than the reference drug nyastatin or, as in the case of compounds $\mathbf{5 b}, \mathbf{6 a}, \mathbf{6 b}, \mathbf{6 d}, \mathbf{6 e}$ and $\mathbf{6 i}$, were inactive against $C$. albicans strain.

\section{Experimental}

\subsection{General}

Melting points were determined in open glass capillaries using in Electrothermal IA 9000 Series digital melting point apparatus (Electrothermal, Essex, UK) and are uncorrected. Elemental analyses were performed with all final compounds with an Elementar, Vario EL, Microanalytical Unit, National Research Centre, Cairo, Egypt and were in good agreement $( \pm 0.2 \%)$ with the calculated values. The IR spectra (KBr) were recorded on an FT IR-8201 PC spectrophotometer (Shimadzu, Tokyo, Japan). The ${ }^{1} \mathrm{H}$ - and ${ }^{13} \mathrm{C}-\mathrm{NMR}$ spectra were measured with a Jeol $270 \mathrm{MHz}$ spectrometer (FTGNM-EX 270, Tokyo, Japan) in DMSO- $d_{6}$ as solvent. The chemical shifts were recorded relative to TMS. The Mass spectra (EI) were run at $70 \mathrm{eV}$ with a Finnegan SSQ 7000 spectrometer (Thermo Instrument System Inc., Madison, WI, USA), $m / z$ values are indicated in Dalton. TLC (silica gel, aluminum sheets $60 \mathrm{~F}_{254}$, Merck, Darmstadt, Germany) was used for tracing the reactions. The starting material 3 was prepared according to the reported procedure [13] (Scheme 1). Antimicrobial screening was carried out in Department of Microbial Chemistry, National Research Center, Cairo, Egypt.

\subsection{Chemistry}

Synthesis of Bisimidotricyclo-[3,23,1,1 $\left.1^{11,15}\right]$ triaconta-1(28),11,13,15,25,27-hexene Derivatives 4 and 5a,b

A suspension of the hydrazide derivative $3(0.554 \mathrm{~g}, 1 \mathrm{mmol})$ and 1,8-naphthalindicarboxylic anhydride, phthalic anhydride or 2,3,4,5-tetrachlorophthalic anhydride (2 mmol) in acetic acid (50 $\mathrm{mL})$ was refluxed for $7 \mathrm{~h}$. The solid was collected by filtration, washed with acetic acid and crystallized from dimethylformamide/water to give the corresponding macrocyclic octaamidodipyridyl derivatives 4 and $\mathbf{5 a}, \mathbf{b}$, respectively.

4,20-Di[oxo-2-(amino-3a,6-dihydro-1H-benzo[de]isoquinoline-1,3(2H)-dione)methyl]-3,8,16,21,27, 28-hexaaza-2,9,15,22-tetraoxotricyclo[3,21,1,1 10,14]octacosa-1(26),10,12,14,22,25-hexene (4): IR $\left(\mathrm{KBr}, \mathrm{cm}^{-1}\right): v 3340\left(\mathrm{NH}\right.$, amide), $1640(\mathrm{C}=\mathrm{N}), 1660,1534,1320$ (amide I, II and III). ${ }^{1} \mathrm{H}-\mathrm{NMR}$ (DMSO-d $)_{6}$ : $\delta 1.32-1.36\left(\mathrm{~m}, 4 \mathrm{H}, 2 \times \mathrm{CH}_{2}\right), 1.54-1.62\left(\mathrm{~m}, 4 \mathrm{H}, 2 \times \mathrm{CH}_{2}\right), 3.18-3.26\left(\mathrm{~m}, 4 \mathrm{H}, 2 \times \mathrm{CH}_{2}\right)$, 4.38-4.54 (m, 2H, $2 \times \mathrm{CH}-\mathrm{N}), 7.75-8.10(\mathrm{~m}, 12 \mathrm{H}, \mathrm{Ar}-\mathrm{H}), 8.24-8.36(\mathrm{~m}, 6 \mathrm{H}, 2 \times$ pyr- $H), 8.92(\mathrm{~m}, 2 \mathrm{H}$, $2 \times \mathrm{N} H$, exchangeable with $\left.\mathrm{D}_{2} \mathrm{O}\right), 9.16\left(\mathrm{~m}, 2 \mathrm{H}, 2 \times \mathrm{N} H\right.$, exchangeable with $\left.\mathrm{D}_{2} \mathrm{O}\right)$ and $10.08(\mathrm{~m}, 2 \mathrm{H}$,

$2 \times \mathrm{NH}$, exchangeable with $\left.\mathrm{D}_{2} \mathrm{O}\right) .{ }^{13} \mathrm{C}-\mathrm{NMR}: 27.62,30.54,38.54\left(6 \mathrm{CH}_{2}\right), 52.26(2 \mathrm{CHNH}), 125.10$, 
$125.16,137.10,137.14,148.10,148.24$ (10pyr-C), 122.45, 124.98, 127.24, 128.95, 137.43, 137.76 (20Ar-C), 163.46, 169.50 (4CONH), 157.88 (4CO-imide), 170.38 (2CO-amide). MS, m/z (\%): 914 [M $\left.\mathrm{M}^{+}, 24\right], 703$ (14), 675 (45), 464 (72), 436 (35), 303 (76), 239 (100); Elemental analysis: calcd. for $\mathrm{C}_{48} \mathrm{H}_{38} \mathrm{~N}_{10} \mathrm{O}_{10}$ (914.87): C, 63.02; H, 4.19; N, 15.31. found: C, 62.85; H, 4.14; N, 15.25.

4,20-Di[oxo-2-(amino-1H-isoindole-1,3(2H)-dione)methyl]-3,8,16,21,27,28-hexaaza-2,9, 15,22-tetraoxotricyclo[3,21,1,1 $\left.1^{10,14}\right]$ octacosa-1(26),10,12,14,22,25-hexene (5a): IR $\left(\mathrm{KBr}, \mathrm{cm}^{-1}\right)$ : v $3338(\mathrm{NH}$, amide), $1642(\mathrm{C}=\mathrm{N}), 1665,1540,1322$ (amide I, II and III). ${ }^{1} \mathrm{H}-\mathrm{NMR}$ (DMSO- $\left.d_{6}\right): \delta 1.23-1.32(\mathrm{~m}$, $\left.4 \mathrm{H}, 2 \times \mathrm{CH}_{2}\right), 1.48-1.58\left(\mathrm{~m}, 4 \mathrm{H}, 2 \times \mathrm{CH}_{2}\right), 3.15-3.20\left(\mathrm{~m}, 4 \mathrm{H}, 2 \times \mathrm{CH}_{2}\right), 4.42-4.52(\mathrm{~m}, 2 \mathrm{H}, 2 \times \mathrm{CH}-\mathrm{N})$, 7.65-7.80 (m, 8H, Ar-H), 8.30-8.38 (m, 6H, $2 \times$ pyr- $H), 8.88(\mathrm{~m}, 2 \mathrm{H}, 2 \times \mathrm{NH}$, exchangeable with $\left.\mathrm{D}_{2} \mathrm{O}\right), 9.12\left(\mathrm{~m}, 2 \mathrm{H}, 2 \times \mathrm{N} H\right.$, exchangeable with $\left.\mathrm{D}_{2} \mathrm{O}\right)$ and $10.15(\mathrm{~m}, 2 \mathrm{H}, 2 \times \mathrm{N} H$, exchangeable with $\left.\mathrm{D}_{2} \mathrm{O}\right) .{ }^{13} \mathrm{C}-\mathrm{NMR}: 27.65,30.52,38.58\left(6 \mathrm{CH}_{2}\right), 52.12(2 \mathrm{CHNH}), 125.08,125.12,137.10,137.18,148.16$, 148.32 (10pyr-C), 123.18, 131.78, 132.45 (12Ar-C), 163.62, 169.58 (4CONH), 164.35 (4CO-imide), 170.15 (2CO-amide). MS, $m / z$ (\%): 815 [M $\left.\mathrm{M}^{+}, 33\right], 653$ (22), 522 (62), 492 (42), 464 (55), 436 (24), 189 (100); Elemental analysis: calcd. for $\mathrm{C}_{40} \mathrm{H}_{34} \mathrm{~N}_{10} \mathrm{O}_{10}$ (814.75): C, 58.97; H, 4.21; N, 17.19. found: C, 58.92; $\mathrm{H}, 4.05 ; \mathrm{N}, 17.15$.

4,20-Di[oxo-2-(amino-4,5,6, 7-tetrachloro-1H-isoindole-1,3(2H)-dione)methyl]-3,8,16,21,27,28-hexaaza-2,9,15,22-tetraoxotricyclo[3,21,1,1 $\left.{ }^{10,14}\right]$ octacosa-1(26), 10,12,14,22,25-hexene (5b): IR $(\mathrm{KBr}$, $\left.\mathrm{cm}^{-1}\right): \vee 3346\left(\mathrm{NH}\right.$, amide), $1645(\mathrm{C}=\mathrm{N}), 1662,1538,1318$ (amide I, II and III). ${ }^{1} \mathrm{H}-\mathrm{NMR}$ (DMSO- $\left.d_{6}\right):$ $\delta 1.26-1.33\left(\mathrm{~m}, 4 \mathrm{H}, 2 \times \mathrm{CH}_{2}\right), 1.38-1.55\left(\mathrm{~m}, 4 \mathrm{H}, 2 \times \mathrm{CH}_{2}\right), 3.18-3.24\left(\mathrm{~m}, 4 \mathrm{H}, 2 \times \mathrm{CH}_{2}\right), 4.46-4.54$ $(\mathrm{m}, 2 \mathrm{H}, 2 \times \mathrm{CH}-\mathrm{N}), 8.26-8.34(\mathrm{~m}, 6 \mathrm{H}, 2 \times$ pyr- $H), 8.92\left(\mathrm{~m}, 2 \mathrm{H}, 2 \times \mathrm{NH}\right.$, exchangeable with $\left.\mathrm{D}_{2} \mathrm{O}\right)$, $9.18\left(\mathrm{~m}, 2 \mathrm{H}, 2 \times \mathrm{NH}\right.$, exchangeable with $\left.\mathrm{D}_{2} \mathrm{O}\right)$ and $10.08\left(\mathrm{~m}, 2 \mathrm{H}, 2 \times \mathrm{NH}\right.$, exchangeable with $\left.\mathrm{D}_{2} \mathrm{O}\right)$. ${ }^{13} \mathrm{C}-\mathrm{NMR}: 27.55,30.58,38.60\left(6 \mathrm{CH}_{2}\right), 52.22(2 \mathrm{CHNH}), 124.98,125.05,137.12,137.16,148.22,148.30$ (10pyr-C), 127.12, 132.96, 134.75 (12Ar-C), 163.58, 169.54 (4CONH), 164.48 (4CO-imide), 170.26 (2CO-amide). MS, m/z (\%):1090 [M+, 8], 789 (15), 492 (64), 464 (32), 436 (24), 324 (100); Elemental analysis: calcd. for $\mathrm{C}_{40} \mathrm{H}_{26} \mathrm{Cl}_{8} \mathrm{~N}_{10} \mathrm{O}_{10}$ (1090.31): C, 44.06; $\mathrm{H}, 2.40 ; \mathrm{Cl}, 26.01 ; \mathrm{N}, 12.85$. found: C, $44.00 ; \mathrm{H}, 2.34 ; \mathrm{Cl}, 25.94 ; \mathrm{N}, 12.80$.

Synthesis of 4,20-Di[oxo-(substituted phenyl)-carbohydrazonylmethyl)-3,8,16,21,27,28-hexaaza-2,9, 15,22-tetraoxotricyclo-[3,21,1,1 $\left.{ }^{10,14}\right]$ octacosa-1(26),10,12,14,23,25-hexenes $\mathbf{6 a - j}$

A mixture of the hydrazide derivative $3(0.554 \mathrm{~g}, 1 \mathrm{mmol})$ and the appropriate aldehyde $(2 \mathrm{mmol})$ in absolute ethanol $(50 \mathrm{~mL})$ was heated under reflux for $6 \mathrm{~h}$. The solvent was evaporated under reduced pressure and the residue was solidified with ether. The solid was collected by filtration, washed with ether and crystallized from a proper solvent to afford the corresponding tricyclohexaazaoctacosabis- hydrazone derivatives $\mathbf{6 a}-\mathbf{j}$, respectively.

4,20-Di[oxphenylcarbohydrazonylmethyl)-3,8,16,21,27,28-hexaaza-2,9,15,22-tetraoxotricyclo-[3,21, $1,1^{10,14}$ ]octacosa-1(26), 10,12,14,23,25-hexene (6a): IR $\left(\mathrm{KBr}, \mathrm{cm}^{-1}\right): v 3342(\mathrm{NH}$, amide $), 1646(\mathrm{C}=\mathrm{N})$, 1668, 1542, 1318 (amide I, II and III). ${ }^{1} \mathrm{H}-\mathrm{NMR}$ (DMSO- $\left.d_{6}\right): \delta 1.28-1.34\left(\mathrm{~m}, 4 \mathrm{H}, 2 \times \mathrm{CH}_{2}\right), 1.50-1.60$ $\left(\mathrm{m}, 4 \mathrm{H}, 2 \times \mathrm{CH}_{2}\right), 3.10-3.18\left(\mathrm{~m}, 4 \mathrm{H}, 2 \times \mathrm{CH}_{2}\right), 4.44-4.56(\mathrm{~m}, 2 \mathrm{H}, 2 \times \mathrm{CH}-\mathrm{N}), 7.45-7.68(\mathrm{~m}, 12 \mathrm{H}$, $2 \mathrm{Ph}-H+2 \mathrm{CH}=\mathrm{N}), 8.25-8.38(\mathrm{~m}, 6 \mathrm{H}, 2 \times$ pyr $-H), 8.86\left(\mathrm{~m}, 2 \mathrm{H}, 2 \times \mathrm{N} H\right.$, exchangeable with $\left.\mathrm{D}_{2} \mathrm{O}\right), 8.94$ 
$\left(\mathrm{m}, 2 \mathrm{H}, 2 \times \mathrm{NH}\right.$, exchangeable with $\left.\mathrm{D}_{2} \mathrm{O}\right)$ and $10.08\left(\mathrm{~m}, 2 \mathrm{H}, 2 \times \mathrm{NH}\right.$, exchangeable with $\left.\mathrm{D}_{2} \mathrm{O}\right)$. ${ }^{13} \mathrm{C}-\mathrm{NMR}: 27.82,30.40,38.52\left(6 \mathrm{CH}_{2}\right), 52.05(2 \mathrm{CHNH}), 147.12(2 \mathrm{CH}=\mathrm{N}), 125.16,125.24,137.15$, 137.22, 148.20, 148.42 (10pyr-C), 123.85, 127.94, 129.38, 132.46 (12Ar-C), 163.66, 169.64 (4CONH), 171.98 (2CO-hydrazone). MS, m/z (\%):730 [M+, 6], 611 (34), 492 (45), 436 (55), 218 (100); Elemental analysis: calcd. for $\mathrm{C}_{38} \mathrm{H}_{38} \mathrm{~N}_{10} \mathrm{O}_{6}$ (730.77): C, 62.46; H, 5.24; N, 19.17. found: $\mathrm{C}, 62.40 ; \mathrm{H}$, 5.17; N, 19.13 .

4,20-Di[oxo-3-bromophenylcarbohydrazonylmethyl)-3,8,16,21,27,28-hexaaza-2,9,15,22-tetraoxotricyclo[3,21,1,1 10,14] octacosa-1(26),10,12,14,23,25-hexene (6b): IR (KBr, $\left.\mathrm{cm}^{-1}\right): v 3343$ (NH, amide), $1640(\mathrm{C}=\mathrm{N}), 1660,1542,1318$ (amide I, II and III). ${ }^{1} \mathrm{H}-\mathrm{NMR}$ (DMSO- $\left.d_{6}\right): \delta 1.26-1.34\left(\mathrm{~m}, 4 \mathrm{H}, 2 \times \mathrm{CH}_{2}\right)$, 1.50-1.62 (m, 4H, $\left.2 \times \mathrm{CH}_{2}\right), 3.18-3.24\left(\mathrm{~m}, 4 \mathrm{H}, 2 \times \mathrm{CH}_{2}\right), 4.50-4.56(\mathrm{~m}, 2 \mathrm{H}, 2 \times \mathrm{CH}-\mathrm{N}), 7.35-7.66(\mathrm{~m}$, $6 \mathrm{H}, \operatorname{Ar}-H), 7.78(\mathrm{~s}, 2 \mathrm{H}, \mathrm{Ar}-\mathrm{H}), 7.92(\mathrm{~s}, 2 \mathrm{H}, 2 \mathrm{CH}=\mathrm{N}), 8.24-8.35(\mathrm{~m}, 6 \mathrm{H}, 2 \times$ pyr- $H), 8.96(\mathrm{~m}, 2 \mathrm{H}, 2 \times \mathrm{N} H$, exchangeable with $\left.\mathrm{D}_{2} \mathrm{O}\right), 9.15\left(\mathrm{~m}, 2 \mathrm{H}, 2 \times \mathrm{N} H\right.$, exchangeable with $\left.\mathrm{D}_{2} \mathrm{O}\right)$ and $10.16(\mathrm{~m}, 2 \mathrm{H}, 2 \times \mathrm{N} H$, exchangeable with $\left.\mathrm{D}_{2} \mathrm{O}\right) .{ }^{13} \mathrm{C}-\mathrm{NMR}: 27.70,30.38,38.52\left(6 \mathrm{CH}_{2}\right), 51.98(2 \mathrm{CHNH}), 147.08(2 \mathrm{CH}=\mathrm{N})$, 125.06, 125.12, 137.10, 137.16, 148.08, 148.16 (10pyr-C), 123.82, 127.94, 129.15, 132.18, 133.82, 135.32 (12Ar-C), 163.68, 169.76 (4CONH), 172.14 (2CO-hydrazone). MS, $m / z(\%): 888$ [M $\left.{ }^{+}, 23\right], 691$ (45), 689 (76), 492 (100), 436 (55), 218 (82); Elemental analysis: calcd. for $\mathrm{C}_{38} \mathrm{H}_{36} \mathrm{Br}_{2} \mathrm{~N}_{10} \mathrm{O}_{6}(888.56)$ : C, 51.36; H, 4.08; N, 15.76. found: C, 51.30; H, 4.00; N, 15.71 .

4,20-Di[oxo-4-bromophenylcarbohydrazonylmethyl)-3,8,16,21,27,28-hexaaza-2,9,15,22-tetraoxotricyclo[3,21,1,1 10,14] octacosa-1(26),10,12,14,23,25-hexene (6c): IR $\left(\mathrm{KBr}, \mathrm{cm}^{-1}\right): v 3338(\mathrm{NH}$, amide), $1644(\mathrm{C}=\mathrm{N}), 1663,1545,1322$ (amide I, II and III). ${ }^{1} \mathrm{H}-\mathrm{NMR}\left(\mathrm{DMSO}_{\mathrm{d}}\right): \delta 1.25-1.35\left(\mathrm{~m}, 4 \mathrm{H}, 2 \times \mathrm{CH}_{2}\right)$, 1.52-1.64 (m, 4H, $\left.2 \times \mathrm{CH}_{2}\right), 3.15-3.20\left(\mathrm{~m}, 4 \mathrm{H}, 2 \times \mathrm{CH}_{2}\right), 4.54-4.58(\mathrm{~m}, 2 \mathrm{H}, 2 \times \mathrm{CH}-\mathrm{N}), 7.55-7.78(\mathrm{~m}$, $10 \mathrm{H}, 2 \mathrm{Ph}-H+2 \mathrm{CH}=\mathrm{N}), 8.18-8.35(\mathrm{~m}, 6 \mathrm{H}, 2 \times$ pyr- $H), 8.88(\mathrm{~m}, 2 \mathrm{H}, 2 \times \mathrm{N} H$, exchangeable with $\left.\mathrm{D}_{2} \mathrm{O}\right), 8.96\left(\mathrm{~m}, 2 \mathrm{H}, 2 \times \mathrm{NH}\right.$, exchangeable with $\left.\mathrm{D}_{2} \mathrm{O}\right)$ and $10.12(\mathrm{~m}, 2 \mathrm{H}, 2 \times \mathrm{NH}$, exchangeable with $\left.\mathrm{D}_{2} \mathrm{O}\right) .{ }^{13} \mathrm{C}-\mathrm{NMR}: 27.75,30.42,38.55\left(6 \mathrm{CH}_{2}\right), 52.15(2 \mathrm{CHNH}), 146.98(2 \mathrm{CH}=\mathrm{N}), 125.18,125.22$, 137.05, 137.12, 148.18, 148.25 (10pyr-C), 123.80, 127.96, 129.34, 133.48 (12Ar-C), 163.65, 169.72 (4CONH), 172.08 (2CO-hydrazone). MS, m/z (\%): 888 [M $\left.\mathrm{M}^{+}, 12\right], 691$ (34), 689 (32), 492 (100), 436 (35), 218 (94); Elemental analysis: calcd. for $\mathrm{C}_{38} \mathrm{H}_{36} \mathrm{Br}_{2} \mathrm{~N}_{10} \mathrm{O}_{6}$ (888.56): C, 51.36; H, 4.08; N, 15.76. found: C, 51.31; H, 4.01; N, 15.72 .

4,20-Di[oxo-2,6-dichlorophenylcarbohydrazonylmethyl)-3,8,16,21,27,28-hexaaza-2,9, 15,22-tetraoxotricyclo[3,21,1,1 10,14]octacosa-1(26),10,12,14,23,25-hexene (6d): IR $\left(\mathrm{KBr}, \mathrm{cm}^{-1}\right): v 3344(\mathrm{NH}$, amide), $1648(\mathrm{C}=\mathrm{N}), 1660,1541,1319$ (amide I, II and III). ${ }^{1} \mathrm{H}-\mathrm{NMR}$ (DMSO-d $\left.)_{6}\right): \delta 1.26-1.32$ (m, $\left.4 \mathrm{H}, 2 \times \mathrm{CH}_{2}\right), 1.50-1.62\left(\mathrm{~m}, 4 \mathrm{H}, 2 \times \mathrm{CH}_{2}\right), 3.18-3.22\left(\mathrm{~m}, 4 \mathrm{H}, 2 \times \mathrm{CH}_{2}\right), 4.52-4.60(\mathrm{~m}, 2 \mathrm{H}, 2 \times \mathrm{CH}-\mathrm{N})$, $7.40-7.48(\mathrm{~m}, 8 \mathrm{H}, 2 \mathrm{Ph}-H+2 \mathrm{CH}=\mathrm{N}), 8.18-8.32(\mathrm{~m}, 6 \mathrm{H}, 2 \times \operatorname{pyr}-H), 8.78(\mathrm{~m}, 2 \mathrm{H}, 2 \times \mathrm{N} H$, exchangeable with $\left.\mathrm{D}_{2} \mathrm{O}\right), 8.98\left(\mathrm{~m}, 2 \mathrm{H}, 2 \times \mathrm{N} H\right.$, exchangeable with $\left.\mathrm{D}_{2} \mathrm{O}\right)$ and $10.15(\mathrm{~m}, 2 \mathrm{H}, 2 \times \mathrm{N} H$, exchangeable with $\left.\mathrm{D}_{2} \mathrm{O}\right) .{ }^{13} \mathrm{C}-\mathrm{NMR}: 27.66,30.39,38.65\left(6 \mathrm{CH}_{2}\right), 52.09(2 \mathrm{CHNH}), 147.068(2 \mathrm{CH}=\mathrm{N})$, 125.23, 125.25, 137.08, 137.10, 148.16, 148.22 (10pyr-C), 126.56, 127.48, 129.36, 133.52 (12Ar-C), 163.58, $169.68(4 C O \mathrm{NH}), 172.15$ (2CO-hydrazone). MS, $m / z(\%): 868\left[\mathrm{M}^{+}, 8\right], 679$ (18), 492 (58), 436 (42), 245 (100), 205 (78); Elemental analysis: calcd. for $\mathrm{C}_{38} \mathrm{H}_{34} \mathrm{Cl}_{4} \mathrm{~N}_{10} \mathrm{O}_{6}$ (868.55): C, 52.55; $\mathrm{H}$, 3.95; Cl, 16.33; N, 16.13. found: C, 52.50; H, 3.91; Cl, 16.28; N, 16.08. 
4,20-Di[oxo-3,4-dichlorophenylcarbohydrazonylmethyl)-3,8,16,21,27,28-hexaaza-2,9, 15,22-tetraoxotricyclo[3,21,1,1 $\left.{ }^{10,14}\right]$ octacosa-1(26), 10,12,14,23,25-hexene (6e): $\mathrm{IR}\left(\mathrm{KBr}, \mathrm{cm}^{-1}\right): v 3346(\mathrm{NH}$, amide), $1626(\mathrm{C}=\mathrm{N}), 1662,1539,1322$ (amide I, II and III). ${ }^{1} \mathrm{H}-\mathrm{NMR}$ (DMSO- $\left.d_{6}\right): \delta 1.28-1.35\left(\mathrm{~m}, 4 \mathrm{H}, 2 \times \mathrm{CH}_{2}\right)$, 1.49-1.60 (m, 4H, $\left.2 \times \mathrm{CH}_{2}\right), 3.24-3.26\left(\mathrm{~m}, 4 \mathrm{H}, 2 \times \mathrm{CH}_{2}\right), 4.46-4.58(\mathrm{~m}, 2 \mathrm{H}, 2 \times \mathrm{CH}-\mathrm{N}), 7.55-7.65(\mathrm{~m}$, $6 \mathrm{H}, 4 \mathrm{H}-\mathrm{Ar}+2 \mathrm{CH}=\mathrm{N}), 7.86(\mathrm{~s}, 2 \mathrm{H}, \mathrm{Ar}-\mathrm{H}), 8.18-8.26(\mathrm{~m}, 6 \mathrm{H}, 2 \times \mathrm{pyr}-H), 8.84(\mathrm{~m}, 2 \mathrm{H}, 2 \times \mathrm{N} H$, exchangeable with $\left.\mathrm{D}_{2} \mathrm{O}\right), 9.05\left(\mathrm{~m}, 2 \mathrm{H}, 2 \times \mathrm{N} H\right.$, exchangeable with $\left.\mathrm{D}_{2} \mathrm{O}\right)$ and $10.18(\mathrm{~m}, 2 \mathrm{H}, 2 \times \mathrm{N} H$, exchangeable with $\left.\mathrm{D}_{2} \mathrm{O}\right) .{ }^{13} \mathrm{C}-\mathrm{NMR}: 27.34,30.42,37.98\left(6 \mathrm{CH}_{2}\right), 51.96(2 \mathrm{CHNH}), 147.08(2 \mathrm{CH}=\mathrm{N})$, $124.95,125.05,137.10,137.14,148.08,148.12$ (10pyr-C), 126.94, 129.45, 129.55, 132.65, 132.76, 134.68 (12Ar-C), 163.45, 169.72 (4CONH), 171.88 (2CO-hydrazone). MS, $m / z(\%): 868$ [M $\left.{ }^{+}, 12\right], 868$ $\left\{\mathrm{M}^{+}+2,5\right], 679$ (22), 492 (25), 436 (56), 245 (78), 214 (100); Elemental analysis: calcd. for $\mathrm{C}_{38} \mathrm{H}_{34} \mathrm{Cl}_{4} \mathrm{~N}_{10} \mathrm{O}_{6}$ (868.55): C, 52.55; H, 3.95; Cl, 16.33; N, 16.13. found: C, 52.48; H, 3.90; Cl, 16.29; $\mathrm{N}, 16.10$.

4,20-Di[oxo-2-chloro-6-fluorophenylcarbohydrazonylmethyl)-3,8,16,21,27,28-hexaaza-2,9,15,22tetraoxo-tricyclo[3,21,1,1 $\left.1^{10,14}\right]$ octacosa-1(26),10,12,14,23,25-hexene (6f): IR $\left(\mathrm{KBr}, \mathrm{cm}^{-1}\right): v 3352$ $\left.\left(\mathrm{NH} \text {, amide), } 1618(\mathrm{C}=\mathrm{N}), 1660,1538,1324 \text { (amide I, II and III). }{ }^{1} \mathrm{H}-\mathrm{NMR} \text { (DMSO-d }\right)_{6}\right): \delta 1.34-1.38$ (m, 4H, $\left.2 \times \mathrm{CH}_{2}\right), 1.44-1.58\left(\mathrm{~m}, 4 \mathrm{H}, 2 \times \mathrm{CH}_{2}\right), 3.30-3.36\left(\mathrm{~m}, 4 \mathrm{H}, 2 \times \mathrm{CH}_{2}\right), 4.50-4.57(\mathrm{~m}, 2 \mathrm{H}, 2 \times \mathrm{CH}-\mathrm{N})$, 7.45-7.85 (m, 8H, Ar-H + 2CH=N), 8.15-8.30 (m, 6H, $2 \times \operatorname{pyr}-H), 8.86(\mathrm{~m}, 2 \mathrm{H}, 2 \times \mathrm{N} H$, exchangeable with $\left.\mathrm{D}_{2} \mathrm{O}\right), 9.10\left(\mathrm{~m}, 2 \mathrm{H}, 2 \times \mathrm{NH}\right.$, exchangeable with $\left.\mathrm{D}_{2} \mathrm{O}\right)$ and $10.16(\mathrm{~m}, 2 \mathrm{H}, 2 \times \mathrm{N} H$, exchangeable with $\left.\mathrm{D}_{2} \mathrm{O}\right) .{ }^{13} \mathrm{C}-\mathrm{NMR}: 27.54,30.36,37.84\left(6 \mathrm{CH}_{2}\right), 52.04(2 \mathrm{CHNH}), 147.12(2 \mathrm{CH}=\mathrm{N})$, 125.12, 125.16, 137.16, 137.24, 147.96, 148.05 (10pyr-C), 113.68, 117.88, 124.82, 133.52, 134.56, 161.02 (12Ar-C), 163.62, $169.76(4 C O \mathrm{NH}), 171.94$ (2CO-hydrazone). MS, $m / z(\%): 834$ [M $\left.\mathrm{M}^{+}, 17\right]$, $8636\left[\mathrm{M}^{+}+2,6\right], 663$ (18), 492 (15), 464 (8), 245 (62), 199 (100); Elemental analysis: calcd. for $\mathrm{C}_{38} \mathrm{H}_{34} \mathrm{Cl}_{2} \mathrm{~F}_{2} \mathrm{~N}_{10} \mathrm{O}_{6}$ (835.64): C, 54.62; H, 4.10; Cl, 8.49; 16.76. found: C, 54.58; H, 4.05; Cl, 8.43; 16.72.

4,20-Di[oxo-4-methylphenylcarbohydrazonylmethyl)-3,8,16,21,27,28-hexaaza-2,9,15,22-tetraoxotricyclo[3,21,1,1 10,14] octacosa-1(26),10,12,14,23,25-hexene (6g): IR $\left(\mathrm{KBr}, \mathrm{cm}^{-1}\right): v 3340(\mathrm{NH}$, amide), $1638(\mathrm{C}=\mathrm{N}), 1660,1552,1324$ (amide I, II and III). ${ }^{1} \mathrm{H}-\mathrm{NMR}$ (DMSO- $\left.d_{6}\right): \delta 1.32-1.38\left(\mathrm{~m}, 4 \mathrm{H}, 2 \times \mathrm{CH}_{2}\right)$, 1.55-1.65 (m, 4H, $\left.2 \times \mathrm{CH}_{2}\right), 2.25\left(\mathrm{~s}, 6 \mathrm{H}, 2 \times \mathrm{CH}_{3}\right), 3.18-3.24\left(\mathrm{~m}, 4 \mathrm{H}, 2 \times \mathrm{CH}_{2}\right), 4.50-4.60(\mathrm{~m}, 2 \mathrm{H}, 2$ $\times \mathrm{CH}-\mathrm{N}), 7.48-7.85(\mathrm{~m}, 10 \mathrm{H}, 2 \mathrm{Ph}-H+2 \mathrm{CH}=\mathrm{N}), 8.24-8.32(\mathrm{~m}, 6 \mathrm{H}, 2 \times$ pyr- $H), 8.78(\mathrm{~m}, 2 \mathrm{H}, 2 \times \mathrm{N} H$, exchangeable with $\left.\mathrm{D}_{2} \mathrm{O}\right), 8.95\left(\mathrm{~m}, 2 \mathrm{H}, 2 \times \mathrm{N} H\right.$, exchangeable with $\left.\mathrm{D}_{2} \mathrm{O}\right)$ and $10.18(\mathrm{~m}, 2 \mathrm{H}, 2 \times \mathrm{N} H$, exchangeable with $\left.\mathrm{D}_{2} \mathrm{O}\right) .{ }^{13} \mathrm{C}-\mathrm{NMR}: 20.32\left(\mathrm{CH}_{3}\right), 27.45,30.32,38.64\left(6 \mathrm{CH}_{2}\right), 52.18(2 \mathrm{CHNH}), 147.08$ $(2 \mathrm{CH}=\mathrm{N}), 124.96,125.05,137.08,137.10,148.14,148.18$ (10pyr-C), 125.80, 128.05, 129.30, 139.48 (12Ar-C), 163.75, 169.77 (4CONH), 172.15 (2CO-hydrazone). MS, $m / z(\%): 759$ [M , 8], 492 (100), 464 (15), 436 (25), 351 (9), 218 (78); Elemental analysis: calcd. for $\mathrm{C}_{40} \mathrm{H}_{42} \mathrm{~N}_{10} \mathrm{O}_{6}$ (758.82): C, 63.31; H, 5.58; N, 18.46. found: C, 63.26; H, 5.51; N, 18.42 .

4,20-Di[oxo-2-methoxyphenylcarbohydrazonylmethyl)-3,8,16,21,27,28-hexaaza-2,9,15,22-tetraoxotricyclo[3,21,1,1 10,14] octacosa-1(26),10,12,14,23,25-hexene (6h): IR ( $\left.\mathrm{KBr}, \mathrm{cm}^{-1}\right): v 3338(\mathrm{NH}$, amide), $1640(\mathrm{C}=\mathrm{N}), 1662,1552,1320$ (amide I, II and III). ${ }^{1} \mathrm{H}-\mathrm{NMR}$ (DMSO- $\left.d_{6}\right): \delta 1.34-1.38\left(\mathrm{~m}, 4 \mathrm{H}, 2 \times \mathrm{CH}_{2}\right)$, $1.62-1.68\left(\mathrm{~m}, 4 \mathrm{H}, 2 \times \mathrm{CH}_{2}\right), 3.18-3.25\left(\mathrm{~m}, 4 \mathrm{H}, 2 \times \mathrm{CH}_{2}\right), 3.78\left(\mathrm{~s}, 6 \mathrm{H}, 2 \times \mathrm{OCH}_{3}\right), 4.45-4.55(\mathrm{~m}, 2 \mathrm{H}$, $2 \times \mathrm{CH}-\mathrm{N}), 7.36-7.76(\mathrm{~m}, 10 \mathrm{H}, 2 \mathrm{Ph}-\mathrm{H}+2 \mathrm{CH}=\mathrm{N}), 8.20-8.32(\mathrm{~m}, 6 \mathrm{H}, 2 \times$ pyr- $H), 8.88(\mathrm{~m}, 2 \mathrm{H}, 2 \times \mathrm{N} H$, exchangeable with $\left.\mathrm{D}_{2} \mathrm{O}\right), 9.10\left(\mathrm{~m}, 2 \mathrm{H}, 2 \times \mathrm{N} H\right.$, exchangeable with $\left.\mathrm{D}_{2} \mathrm{O}\right)$ and $10.32(\mathrm{~m}, 2 \mathrm{H}, 2 \times \mathrm{N} H$, 
exchangeable with $\left.\mathrm{D}_{2} \mathrm{O}\right) .{ }^{13} \mathrm{C}-\mathrm{NMR}: 27.45,30.68,38.82\left(6 \mathrm{CH}_{2}\right), 52.48(2 \mathrm{CHNH}), 55.14\left(2 \mathrm{C}, 2 \mathrm{OCH}_{3}\right)$, $147.30(2 \mathrm{CH}=\mathrm{N}), 124.86,125.02,136.95,137.04,148.06,148.18(10 \mathrm{pyr}-\mathrm{C}), 112.75,115.86,120.86$, 131.14, 132.05, 156.95 (12Ar-C), 163.68, 170.08 (4CONH), 172.55 (2CO-hydrazone). MS, $\mathrm{m} / z$ (\%):790 [M+, 15], 657 (12), 641 (45), 528 (22), 379 (95), 351 (35), 218 (100), 149 (18); Elemental analysis: calcd. for $\mathrm{C}_{40} \mathrm{H}_{42} \mathrm{~N}_{10} \mathrm{O}_{8}$ (790.82): C, 60.75; H, 5.35; N, 17.71. found: $\mathrm{C}, 60.70 ; \mathrm{H}, 5.30 ; \mathrm{N}, 17.68$.

4,20-Di[oxo-4-methoxyphenylcarbohydrazonylmethyl)-3,8,16,21,27,28-hexaaza-2,9,15,22-tetraoxotricyclo[3,21,1,1 10,14]octacosa-1(26),10,12,14,23,25-hexene (6i): IR ( $\left.\mathrm{KBr}, \mathrm{cm}^{-1}\right): v 3346$ (NH, amide), $1642(\mathrm{C}=\mathrm{N}), 1662,1555,1319$ (amide I, II and III). ${ }^{1} \mathrm{H}-\mathrm{NMR}$ (DMSO- $\left.d_{6}\right): \delta 1.28-1.35\left(\mathrm{~m}, 4 \mathrm{H}, 2 \times \mathrm{CH}_{2}\right)$, 1.60-1.67 (m, 4H, $\left.2 \times \mathrm{CH}_{2}\right), 3.20-3.26\left(\mathrm{~m}, 4 \mathrm{H}, 2 \times \mathrm{CH}_{2}\right), 3.68\left(\mathrm{~s}, 6 \mathrm{H}, 2 \times \mathrm{OCH}_{3}\right), 4.44-4.58(\mathrm{~m}, 2 \mathrm{H}$, $2 \times \mathrm{CH}-\mathrm{N}), 7.58-7.90(\mathrm{~m}, 10 \mathrm{H}, 2 \mathrm{Ph}-H+2 \mathrm{CH}=\mathrm{N}), 8.22-8.30(\mathrm{~m}, 6 \mathrm{H}, 2 \times$ pyr- $H), 8.84(\mathrm{~m}, 2 \mathrm{H}, 2 \times \mathrm{N} H$, exchangeable with $\left.\mathrm{D}_{2} \mathrm{O}\right), 9.06\left(\mathrm{~m}, 2 \mathrm{H}, 2 \times \mathrm{N} H\right.$, exchangeable with $\left.\mathrm{D}_{2} \mathrm{O}\right)$ and $10.24(\mathrm{~m}, 2 \mathrm{H}, 2 \times \mathrm{N} H$, exchangeable with $\left.\mathrm{D}_{2} \mathrm{O}\right) .{ }^{13} \mathrm{C}-\mathrm{NMR}: 27.52,30.62,38.74\left(6 \mathrm{CH}_{2}\right), 52.22(2 \mathrm{CHNH}), 54.66\left(2 \mathrm{C}, 2 \mathrm{OCH}_{3}\right)$, $147.12(2 \mathrm{CH}=\mathrm{N}), 125.05,125.10,137.10,137.14,148.16,148.24(10 \mathrm{pyr}-\mathrm{C}), 113.98,125.64,129.68$, 162.62 (12Ar-C), 163.82, $169.76(4 C O N H), 172.25$ (2CO-hydrazone). MS, $m / z(\%): 790\left[\mathrm{M}^{+}, 24\right], 657$ (9), 641 (76), 528 (12), 379 (100), 351 (45), 218 (78), 149 (68); Elemental analysis: calcd. for $\mathrm{C}_{40} \mathrm{H}_{42} \mathrm{~N}_{10} \mathrm{O}_{8}$ (790.82): C, 60.75; H, 5.35; N, 17.71. found: C, 60.69; H, 5.31; N, 17.67.

4,20-Di[oxo-2,3,5-trimethoxyphenyl-carbohydrazonylmethyl)-3,8,16,21,27,28-hexaaza-2,9,15,22tetraoxotricyclo $\left[3,21,1,1^{10,14}\right]$ octacosa-1(26), 10,12,14,23,25-hexene $(\mathbf{6 j}): \mathrm{IR}\left(\mathrm{KBr}, \mathrm{cm}^{-1}\right): \vee 3336(\mathrm{NH}$, amide), $1640(\mathrm{C}=\mathrm{N}), 1662,1556,1322$ (amide I, II and III). ${ }^{1} \mathrm{H}-\mathrm{NMR}$ (DMSO- $\left.d_{6}\right): \delta 1.32-1.36$ (m, $\left.4 \mathrm{H}, 2 \times \mathrm{CH}_{2}\right), 1.58-1.65\left(\mathrm{~m}, 4 \mathrm{H}, 2 \times \mathrm{CH}_{2}\right), 3.18-3.28\left(\mathrm{~m}, 4 \mathrm{H}, 2 \times \mathrm{CH}_{2}\right), 3.72\left(\mathrm{~s}, 18 \mathrm{H}, 6 \times \mathrm{OCH}_{3}\right)$, 4.34-4.42 (m, 2H, $2 \times \mathrm{CH}-\mathrm{N}), 7.25(\mathrm{~s}, 4 \mathrm{H}, 2 \mathrm{Ph}-\mathrm{H}), 7.88(\mathrm{~s}, 2 \mathrm{H}, 2 \mathrm{CH}=\mathrm{N}), 8.26-8.34(\mathrm{~m}, 6 \mathrm{H}, 2 \times$ pyr- $H)$, $8.92\left(\mathrm{~m}, 2 \mathrm{H}, 2 \times \mathrm{NH}\right.$, exchangeable with $\left.\mathrm{D}_{2} \mathrm{O}\right), 9.14\left(\mathrm{~m}, 2 \mathrm{H}, 2 \times \mathrm{NH}\right.$, exchangeable with $\left.\mathrm{D}_{2} \mathrm{O}\right)$ and $10.16\left(\mathrm{~m}, 2 \mathrm{H}, 2 \times \mathrm{NH}\right.$, exchangeable with $\left.\mathrm{D}_{2} \mathrm{O}\right) \cdot{ }^{13} \mathrm{C}-\mathrm{NMR}: 27.46,30.68,38.66\left(6 \mathrm{CH}_{2}\right), 52.18$ $(2 \mathrm{CHNH}), 55.16\left(4 \mathrm{C}, 4 \mathrm{OCH}_{3}\right), 58.78\left(2 \mathrm{C}, 2 \mathrm{OCH}_{3}\right), 147.24(2 \mathrm{CH}=\mathrm{N}), 124.98,125.02,137.15,137.18$, $148.10,148.14$ (10C, pyr-C), 105.12, 127.35, 140.64, 152.76 (12Ar-C), 163.86, 169.69 (4CONH), 172.22 (2CO-hydrazone). MS, $m / z(\%): 910$ [M $\left.\mathrm{M}^{+}, 15\right], 777$ (8), 701 (12), 673 (25), 692 (76), 436 (58), 303 (22), 237 (100), 218 (78); Elemental analysis: calcd. for $\mathrm{C}_{44} \mathrm{H}_{50} \mathrm{~N}_{10} \mathrm{O}_{12}$ (910.92): C, 58.01; H, 5.53; N, 15.38. found: C, 57.95; H, 5.48; N, 15.33.

\subsection{Antimicrobial Testing}

The antimicrobial activity for the tested compounds was measured at $50 \mu \mathrm{g} / \mathrm{mL}$, and was determined by the agar diffusion method as recommended by the National Committee for Clinical Laboratory Standards (NCCLS) [28-30]. The degree of inhibition is measured in $\mathrm{mm}$ in comparison with that of ampicillin and ketaconazole at the same concentration taken as standard values.

In this method, two agar media, nutrient agar for bacteria and Czapek Dox agar for fungi were prepared and sterilized by autoclaving at $120^{\circ} \mathrm{C}$ and $1.5 \mathrm{~atm}$. for $20 \mathrm{~min}$. The agar plates were poured left to cool down and after solidification they were inoculated with the bacterial and fungal strains by streaking. The tested compounds were loaded on sterile filter paper discs ( $5 \mathrm{~mm}$ diameter) at $50 \mu \mathrm{g} / \mathrm{mL}$ of DMSO and transferred aseptically into the inoculated agar plates along with ampicillin and ketaconazole discs at the same concentration for comparison. The plates were then incubated at $37{ }^{\circ} \mathrm{C}$ 
for $24 \mathrm{~h}$ for bacteria and at $30{ }^{\circ} \mathrm{C}$ for $48-72 \mathrm{~h}$ for fungi. After incubation, the diameters of the inhibition zones around the paper discs were measured in $\mathrm{mm}$ as an indication of the antimicrobial activities of the compounds.

\section{Conclusions}

A series of chiral macrocyclic imides and Schiff-bases 4-6 were synthesized using as starting material the macrocyclic bis-hydrazide derivative 3 [13], obtained from the corresponding ester 2 according to the published procedure $[23,24]$. The structure assignments of the new compounds are based on chemical and spectroscopic evidence. The newly synthesized compounds have been tested for their preliminary antimicrobial activity against Gram-positive bacteria, Gram-negative bacteria, and fungi. From the results it appears that some of the synthesized compounds have modest antimicrobial activities. The activities of all tested compounds against Gram positive bacteria $(B$. subtilis and $S$. aureus) are higher than that of the reference drug except for $\mathbf{6 c}, \mathbf{6} \mathbf{g}$ (against $B$. subtilis) and $\mathbf{6 d}, \mathbf{6 e}, \mathbf{6 h}, \mathbf{6 i}$ against $S$. aureus. The activities of compounds $3, \mathbf{4}, \mathbf{5 a}, \mathbf{b}, \mathbf{6 b}, \mathbf{6 d}$, and $\mathbf{6 g}-\mathbf{j}$ against the Gram negative bacterium E. coli were similar to that of the reference drug, while compounds $\mathbf{5 b}$, $\mathbf{6 a}, \mathbf{6 c}, \mathbf{6 e}$ and $\mathbf{6 f}$ are not active against the same organism. In addition, the compounds which were tested against fungal organisms (C. albicans and A. niger) had lower activities than the reference drug, or, as in the case of compounds $5 \mathbf{b}, \mathbf{6 a}, \mathbf{6 b}, \mathbf{6 d}, \mathbf{6 e}$ and $\mathbf{6 i}$, were not active against the $C$. albicans strain.

\section{Acknowledgements}

The authors extend their appreciation to the Deanship of Scientific Research at King Saud University for funding the work through the research group project No. RGP-VPP-172.

\section{References}

1. Krakowiak, K.E.; Bradshaw, J.S.; Zamecka-Krakowiak, D.J. Synthesis of aza-crown ethers. Chem. Rev. 1989, 89, 929-972.

2. Bradshaw, J.S.; Krakowiak, K.E.; Izatt, R.M. Preparation of diamino ethers and polyamines. Tetrahedron 1992, 48, 4475-4515.

3. Bradshaw, J.S.; Krakowiak, K.E.; Izatt, R.M. Azacrown Macrocycles. In The Chemistry of Heterocyclic Compounds; Taylor, E.C., Ed.; John Wiley \& Sons: New York, NY, USA, 1993; p. 51.

4. Molina, P.; Tarraga, A.; Gaspar, C.; Espinosa, A. Synthesis of a novel class of macrocyclic compounds containing 1,3,4-thiadiazole rings as subunits. J. Org. Chem. 1994, 59, 3665-3669.

5. Izatt, R.M.; Pawlak, K.; Bradshaw, J.S.; Bruening, R.L. Thermodynamic and kinetic data for macrocycle interaction with cations, anions, and neutral molecules. Chem. Rev. 1995, 95, 2529-2586.

6. Izatt, R.M.; Pawlak, K.; Bradshaw, J.S.; Bruening, R.L.; Tarbet, B. Thermodynamic and kinetic data for macrocycle interaction with neutral molecules. J. Chem. Rev. 1992, 92, 1261-1354.

7. Elwahy, A.H.M. New trends in the chemistry of condensed heteromacrocycles Part A: Condensed azacrown ethers and azathiacrown ethers. J. Heterocycl. Chem. 2003, 40, 1-23. 
8. Chu, T.D.W.; Plattner, J.J.; Kotz, L. New directions in antibacterial research. J. Med. Chem. 1996, 39, 3853-3874.

9. Hirschmann, R.; Smith, A.B.; Sprengeler, P.A. New Perspectives in Drug Design; Academic: New York, NY, USA, 1995.

10. Amr, A.E.; Mohamed, A.M.; Ibrahim, A.A. Synthesis of some new chiral tricyclic and macrocyclic pyridine derivatives as antimicrobial agents. Z. Naturforsch. 2003, 58b, 861-868.

11. Amr, A.E. Synthesis of some new linear and chiral macrocyclic pyridine carbazides as analgesic and anticonvulsant agents. Z. Naturforsch. 2005, 60b, 990-998.

12. Abou-Ghalia, M.H.; Amr, A.E.; Abdalah, M.M. Synthesis of some new ( $\mathrm{N}^{\alpha}$-dipicolinoyl)-bis-Lleucyl-DL-norvalyl linear tetra and cyclic octa bridged peptides as new anti-inflammatory agents. Z. Naturforsch. 2003, 58b, 903-910.

13. Amr, A.E.; Abo-Ghalia, M.H.; Abdalah, M.M. Synthesis of novel macrocyclic peptidocalix[4]arenes and peptidopyridines as precursors for potential molecular metallacages, chemosensors and biologically active candidates. Z. Naturforsch. 2006, 61b, 1335-1345.

14. Amr, A.E.; Abo-Ghalia, M.H.; Abdalah, M.M. Synthesis of new (Na-dipicolinoyl)-bis-L-valyl-Lphenylalanyl linear and macrocyclic bridged peptides as anti-inflammatory agents. Arch. Pharm. Chem. Life Sci. 2007, 340, 304-309.

15. Hassan, S.S.M.; Abo-Ghalia, M.H.; Amr, A.E.; Mohamed, A.H.K. New lead (II) selective membrane potentiometric sensors based on chiral 2,6-bis-pyridinecarboximide derivatives. Talanta 2003, 60, 81-91.

16. Hassan, S.S.M.; Abo-Ghalia, M.H.; Amr, A.E.; Mohamed, A.H.K. Novel thiocyanate-selective membrane sensors based on di-, tetra-, and hexaimidopyridine ionophores. Anal. Chem. Acta 2003, 482, 9-18.

17. Al-Mohizea, A.M.; Al-Omar, M.A.; Abdalla, M.M.; Amr, A.E. 5 $\alpha$-Reductase inhibitors, antiviral and anti-tumor activities of some steroidal cyanopyridinone derivatives. Int. J. Biol. Macromol. 2012, 50, 171-179.

18. Abdalla, M.M.; Al-Omar, M.A.; Bhat, M.A.; Amr, A.E.; Al-Mohizea, A.M. Steroidal pyrazolines evaluated as aromatase and quinone reductase- 2 inhibitors for chemoprevention of cancer. Int. J. Biol. Macromol. 2012, 50, 1127-1132.

19. Fakhr, I.M.; Amr, A.E.; Sabry, N.M.; Abdalah, M.M. Anti-inflammatory and analgesic activities of newly synthesized chiral peptide derivatives using (3-benzoyl-4,5-dioxo-2-phenyl-pyrrolidin1-yl)acetic acid ethyl ester ass material. Arch. Pharm. Chem. Life Sci. 2008, 341, 174-180.

20. Amr, A.E. Anticonvulsant and antiparkinsonian evaluation of some synthesized chiral peptide derivatives using 3-benzoyl-4,5-dioxo-2-phenylpyrrolidin-1-yl acetic acid. World J. Chem. 2010, 5, $1-6$.

21. Al-Salahi, R.A.; Al-Omar, M.A.; Amr, A.E. Synthesis of chiral macrocyclic or linear pyridine carboxamides from pyridine-2,6-dicarbonyl dichloride as antimicrobial agents. Molecules 2010, $15,6588-6597$.

22. Al-Omar, M.A.; Amr, A.E. Synthesis of some new pyridine-2,6-carboxamide-derived Schiff bases as potential antimicrobial agents. Molecules 2010, 15, 4711-4721.

23. Attia, A.; Abdel-Salam, O.I.; Amr, A.E.; Stibor, I.; Budesinsky, M. Synthesis and antimicrobial activity of some new chiral bridged macrocyclic pyridines. Egypt. J. Chem. 2000, 43, 187-201. 
24. Amr, A.E.; Abdel-Salam, O.I.; Attia, A.; Stibor, I. Synthesis of new potential bis-intercallators based on chiral pyridine-2,6-dicarbox-amides. Collect. Czech. Chem. Commun. 1999, 64, 288-298.

25. Boxworth, D.M. The treatment of tuberculous lesions of bones and joints with iproniazid (marsilid). N. Y. State J. Med. 1956, 56, 1281-1284.

26. Schroder, H.; Lubke, K. Synthesis, Occurrence and Action of Biologically Active Peptides, Volume II, Translated by Erhard Gross; Academic Press: New York, NY, USA and London, UK, 1966; p. 405.

27. Rollas, S.; Küçükgüzel, S.G. Biological activities of hydrazone derivatives. Molecules 2007, 12, 1910-1939.

28. National Committee for Clinical Laboratory for Standards (NCCLS). Performance Standards for Antimicrobial Testing, Eighth information supplement; Publication No. NCCLS M 100-58; NCCLS: Villanova, PA, USA, 1998.

29. National Committee for Clinical Laboratory for Standards (NCCLS). Methods for Determining Bactericidal Activity of Antimicrobial Agents, Tentative guidelines; Publication No. NCCLS M26-T; NCCLS: Villanova, PA, USA, 1992.

30. Furtado, G.L.; Medeiros, A.A. Single-disk diffusion testing (Kirby-Bauer) of susceptibility of Proteus mirabilis to chloramphenicol: Significance of the intermediate category. J. Clin. Microbiol. 1980, 12, 550-553.

Sample Availability: Samples of the compounds are available from the authors.

(C) 2012 by the authors; licensee MDPI, Basel, Switzerland. This article is an open access article distributed under the terms and conditions of the Creative Commons Attribution license (http://creativecommons.org/licenses/by/3.0/). 